\title{
Post-conflict slowing: cognitive adaptation after conflict processing
}

\author{
Tom Verguts • Wim Notebaert • Wilfried Kunde • \\ Peter Wühr
}

Published online: 5 November 2010

(C) Psychonomic Society, Inc. 2010

\begin{abstract}
The aftereffects of error and conflict (i.e., stimulus or response incongruency) have been extensively studied in the cognitive control literature. Each has been characterized by its own behavioral signature on the following trial. Conflict leads to a reduced congruency effect (Gratton effect), whereas an error leads to increased response time (post-error slowing). The reason for this dissociation has remained unclear. Here, we show that post-conflict slowing is not typically observed because it is masked by the processing of the irrelevant stimulus dimension. We demonstrate that post-conflict slowing does occur when tested in pure trials where helpful or detrimental impacts from irrelevant stimulus dimensions are removed (i.e., univalent stimuli).
\end{abstract}

Keywords Cognitive control · Post-error slowing · Simon task

Carrying out a cognitive task does not always proceed smoothly. Think for example about driving through the woods when suddenly a deer jumps in front of your car. Such problems can be useful in informing us about relevant task constraints and characteristics: In the example, one might learn to drive carefully when there is a chance that animals are hiding behind the trees.

There is a recent interest in the literature on how task processing changes after such problems (e.g., Botvinick,

T. Verguts $(\bowtie) \cdot$ W. Notebaert

Department of Psychology, Ghent University,

H. Dunantlaan 2,

9000 Ghent, Belgium

e-mail: tom.verguts@ugent.be

W. Kunde $\cdot$ P. Wühr

Institut für Psychologie, Technische Universität Dortmund,

Dortmund, Germany
Braver, Barch, Carter, \& Cohen, 2001). One obvious type of problem is an error, where an inappropriate response is given. There are also more subtle problems, for instance, when different stimulus aspects point toward different responses. To continue our example, the road demarcation suggests keeping driving in the same track, whereas the jumping deer suggests switching lanes. This type of conflict is studied with congruency tasks using stimuli that have both a relevant and an irrelevant processing dimension (bivalent stimuli; e.g., Simon and Stroop tasks). When the two dimensions point toward the same response (e.g., in the Stroop task, stimulus RED in red ink), the stimulus is congruent; otherwise (e.g., RED in green ink), it is incongruent. The relevant dependent variable is the congruency effect, the difference (e.g., in response time (RT)) between congruent and incongruent trials.

Both types of problems have been characterized by a different signature on subsequent task processing. An incongruency is typically followed by a reduced congruency effect on the next trial (Gratton effect; see Gratton, Coles, \& Donchin, 1992). An influential framework to account for this Gratton effect is the conflict monitoring theory of Botvinick and colleagues (Botvinick et al., 2001). According to this theory, incongruency leads to response conflict, meaning the simultaneous activation of two or more response alternatives (see Botvinick et al. for formal definition of response conflict). Detection of response conflict leads to an increased focusing on the task-relevant stimulus properties on the next trial. This process will be called task focusing. As a consequence, the irrelevant dimension has less influence on the next trial, thus decreasing the congruency effect.

After errors, on the other hand, participants typically slow down (post-error slowing; Rabbitt \& Phillips, 1967). Also post-error slowing has been interpreted within the 
framework of conflict monitoring theory. In particular, error is correlated with response conflict, in that error trials also typically lead to high response conflict (i.e., simultaneous activation of correct and incorrect responses). The issue of fast errors in which a response is given with very little activation buildup is beyond the scope of the current paper so we do not consider it further. Botvinick and colleagues argued that when response conflict is detected on error trials, the baseline activation of responses is decreased. As a result, one would become more cautious and slower. This same mechanism, however, might also be usefully employed for incongruent trials, which also lead to response conflict. Indeed, if the stimulus set contains many incongruent stimuli, it seems wise to respond cautiously. We will refer to slowing after an incongruent stimulus as post-conflict slowing. Such post-conflict slowing, however, is usually not observed (e.g., Kerns et al., 2004; Notebaert \& Verguts, 2008; but see Ullsperger, Bylsma, \& Botvinick, 2005 who obtained a trend toward post-conflict slowing). This is even more surprising given that there is a research literature on the bivalency effect where slowing does occur because of conflict on earlier trials (e.g., Masson, Bub, Woodward, \& Chan, 2003; Meier, Woodward, ReyMermet, \& Graf, 2009). In this paradigm, subjects predictably switch between different tasks (e.g., color naming, parity judgment, case judgment). In some blocks, stimuli on one task (e.g., case judgment) are bivalent in that they allow two tasks to be performed, possibly leading to a different response (in our terminology, they can be congruent or incongruent). Here, slowing is observed on univalent stimuli in blocks containing bivalent stimuli relative to the same univalent stimuli embedded in blocks containing only univalent stimuli.

To explain this absence of post-conflict slowing in typical cognitive control studies (e.g., Kerns et al., 2004) we think it is crucial to note that the effect of incongruency on slowing in the next trial is typically tested in trials which themselves are congruent or incongruent, that is, bivalent. This is not an ideal situation to evaluate post-conflict slowing because post-conflict focusing will operate maximally also in this case, and this may mask post-conflict slowing. In a congruent trial, because both stimulus dimensions point to the same response, an increased attention to the relevant dimension (post-conflict focusing) will not greatly change the performance. However, an incongruent trial will be strongly influenced by postconflict focusing, because attention on the relevant dimension will improve task processing. As a consequence, the increased RT due to post-conflict slowing will be counteracted on incongruent trials due to post-conflict focusing, thus masking the post-conflict slowing component. To test for post-conflict slowing it would therefore be desirable to use univalent post-conflict probe trials where there is neither helpful nor detrimental impact from irrelevant stimulus dimensions. We therefore used a design consisting of bivalent prime Simon trials (e.g., relevant color and irrelevant location) and either bivalent (same as prime trials) or univalent (color or location only) probe trials. On bivalent probe trials, we expect a reduced Simon effect after incongruent trials but no overall slowing, replicating earlier cognitive control literature. On univalent probe trials, on the other hand, we expect post-conflict slowing.

\section{Method}

\section{Participants}

In experiment 1,18 volunteers (ten female, eight male; range $20-29$ years) participated for payment $(5 €)$ or course credit. In experiment 2, 20 new volunteers (16 female, four male; range 19-30 years) were recruited. All participants were naive with respect to the purpose of the study.

\section{Procedure}

We presented prime-probe stimulus pairs (see Fig. 1). In experiment 1 , color (green or red, left or right key response) was the relevant dimension in the prime trials and location was the irrelevant dimension that makes a stimulus congruent or incongruent (i.e., Simon task). Prime trials were laterally presented (left, right, up, or down). Spatial congruency between stimulus and response location was varied in three levels (congruent, neutral, incongruent). The effect of congruency in prime trials is tested, first, on probe Simon color trials (same task and stimuli as the prime trials but no neutral condition) to evaluate the Gratton effect. Second, it was also tested on univalent color discrimination trials (no irrelevant stimulus dimension) to evaluate postconflict slowing. In these trials, colors were presented at the central location (which was not used in the Simon task), thus minimizing the need for task focusing in such trials. Third, we also included univalent location discrimination trials in which the irrelevant dimension of the prime trials (location) was relevant. Here, a white square was presented left or right, but participants now responded to its location. The latter condition was included to test whether we were successful in excluding the behavioral consequences of post-conflict task focusing in our univalent discrimination trials. The reasoning was that task focusing predicts an opposite trend for univalent color discrimination trials (i.e., same relevant dimension as in Simon task) than for univalent location discrimination trials (irrelevant dimension in Simon task): If more attention is assigned to the relevant dimension, there should be speeding after conflict if the same dimension remains relevant (univalent color 
trials), but slowing if the previously irrelevant dimension becomes relevant (univalent location trials). Hence, if the (slowing) effect is similar in the two probe types, we know that post-conflict focusing is not operative in these trials.

In each trial, five placeholder frames appeared on the screen and remained alone for $500 \mathrm{~ms}$. Then the relevant stimulus appeared. The stimulus and the frames remained until response onset, or until 1,500 ms had elapsed without a response. Finally, a blank screen was shown for $500 \mathrm{~ms}$. The response-to-stimulus interval (RSI) was $500 \mathrm{~ms}$. An error message (centrally presented for 1,500 ms in yellow) occurred when participants pressed the wrong key within $1,500 \mathrm{~ms}$. When RT was larger than $1,500 \mathrm{~ms}$, the participant was asked to respond faster.

Participants first practiced the color discrimination task (12 trials), then the Simon task (18 trials), then the location discrimination task (ten trials), and finally all tasks together (20 trials). In the test phase, participants worked through 12 blocks of 54 trials each. All prime/probe pairs were randomly intermixed. After each block, participants were informed about the number of remaining blocks and were offered a short break. The experiment took $30 \mathrm{~min}$ on average.

Experiment 2 was similar except that letter shape rather than color was the relevant dimension in the Simon task. The stimuli for the Simon task and for the pure shape discrimination task were the capital letters B, $\mathrm{K}, \mathrm{T}$ (left key response), and $\mathrm{F}, \mathrm{P}$, and $\mathrm{Z}$ (right key response), presented in the same boxes as used in experiment 1.

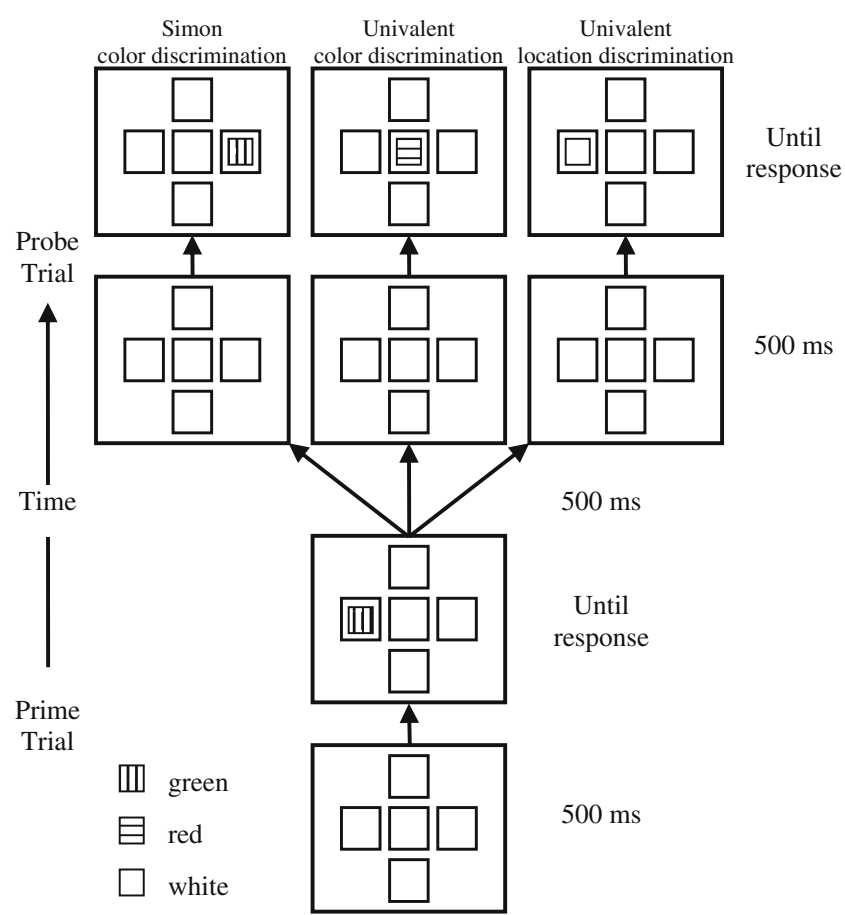

Fig. 1 Possible sequences of stimulus displays in experiment 1
Apparatus and stimuli

Participants sat in a dimly lit room in front of a 17-inch color monitor (viewing distance approximately $60 \mathrm{~cm}$ ). Responses were key presses on a keyboard number pad. Participants operated the left key (\#4) with the index finger and the right key (\#6) with the ring finger of the right hand.

Each placeholder frame was $2 \times 2 \mathrm{~cm}$ and subtended approximately $1.9^{\circ} \times 1.9^{\circ}$ of visual angle. The central frame was presented at fixation, the peripheral frames were presented left, right, above and below fixation. The distance between a peripheral frame and the central frame was $3 \mathrm{~cm}$ $\left(2.9^{\circ}\right)$ from edge to edge. All stimuli were $1 \times 1 \mathrm{~cm}$ squares presented on a black background.

\section{Results}

We removed RTs shorter than $100 \mathrm{~ms}$ or longer than $1,500 \mathrm{~ms}$ (both $<1.0 \%$ ). We only analyzed performance in correct probe trials where also the preceding prime trial was correct.

\section{Experiment 1}

Prime trials RTs and percentage of errors (PE) are reported in Table 1. RTs were subjected to ANOVA with spatial congruency as a within-subjects variable. The effect of this variable was significant, indicative of a Simon effect, $F(2,34)=79.40, M S E=124.78, p<0.001$. RTs were shorter in the congruent than in the neutral condition and longer in the incongruent than in the neutral condition (both $p<0.05)$. ANOVA on PE revealed a similar pattern, $F(2$, $34)=28.21, M S E=8.53, p<0.001$. PE was somewhat smaller in the congruent than in the neutral condition $(p=.05)$, but larger in the incongruent than in the neutral condition $(p<0.001)$.

Probe trials: Simon task RTs from the probe-trial Simon task are shown in Fig. 2a (see Table 2). ANOVA with previous trial (i.e., prime) congruency (congruent, neutral, incongruent) and current-trial (i.e., probe) congruency (congruent, incongruent) as within-subjects variables failed to find a main effect of prime congruency on probe RTs $(F<1)$, replicating the absence of post-conflict slowing in the cognitive control

Table 1 RTs and PEs (in parentheses) in the prime trial Simon tasks

\begin{tabular}{llll}
\hline & \multicolumn{2}{l}{ Spatial congruency } \\
\cline { 2 - 4 } & Congruent & Neutral & Incongruent \\
\hline Experiment 1 & $452(2.1)$ & $487(3.8)$ & $497(9.1)$ \\
Experiment 2 & $601(3.1)$ & $631(3.6)$ & $644(6.2)$ \\
\hline
\end{tabular}


literature (e.g., Kerns et al., 2004). The main effect of probe congruency was significant, $F(1,17)=86.93, M S E=576.26$, $p<0.001$ (Simon effect): RTs were shorter in the congruent than in the incongruent condition. The two-way interaction was also significant, $F(2,34)=31.81, M S E=395.51$, $p<0.001$, indicative of a Gratton effect.

ANOVA on PEs revealed similar results. There was no main effect of prime congruency $(F<1)$, but there was a main effect of probe congruency, $F(1,17)=38.15$, $M S E=7.08, p<0.001(1.7$ vs. $4.9 \%)$. The interaction was also significant, $F(2,34)=6.04, M S E=16.96$, $p<.01$, indicative of a Gratton effect: Simon effects were $5.6,4.6$, and $-0.7 \%$, after congruent, neutral, and incongruent primes, respectively.

Probe trials: univalent color discrimination task RTs are shown in Fig. $2 \mathrm{~b}$. The effect of prime congruency was significant, $F(2,34)=4.54, M S E=245.15, p<0.05$. RTs were similar after congruent and neutral Simon trials, $t(17)=0.86, p=.40$, but longer after incongruent Simon trials than both after neutral and after congruent Simon trials, both $t$ 's $(17)>2.10$, both $p$ 's $<0.05$.

ANOVA on PEs revealed a marginally significant effect, $F(2,34)=3.00, M S E=8.68, p=0.06$, indicating a similar pattern of results as in RTs. In particular, when compared to PEs after congruent $(M=1.7 \%)$ and after neutral Simon trials $(M=1.6 \%)$, PEs increased after incongruent Simon trials $(M=3.7 \%)$.

Probe trials: univalent location discrimination task RTs are shown in Fig. 2b. The effect of prime congruency was significant, $F(2,34)=10.86, M S E=401.26, p<0.001$. RTs were significantly faster after congruent Simon trials than after neutral and incongruent Simon trials, both $t^{\prime} \mathrm{s}(17)>2.50$, both $p$ 's $<0.05$. Moreover, RTs were somewhat slower after incongruent Simon trials than after neutral Simon trials, $t(17)=1.98, p=.06$.

There was a significant effect of prime congruency on PEs, $F(2,34)=3.50, M S E=26.82, p<0.05$; PEs were lower after congruent Simon trials $(8.3 \%)$ than after neutral $(12.5 \%)$ or incongruent trials $(12.1 \%)$.

\section{Experiment 2}

For brevity, some statistical comparisons are omitted, but they are fully consistent with those reported in experiment 1.

Prime trials RTs and PEs are reported in Table 1. RTs were shorter in the congruent than in the neutral condition and RTs were longer in the incongruent than in the neutral condition (both $p$ 's $<0.05$ ). The PE was similar in the congruent and in the neutral condition, $t(19)=1.19$, $p=.25$, but larger in the incongruent than in the neutral condition, $t(19)=3.17, p<0.01$.

Probe trials: Simon task RTs are shown in Fig. 2c (see Table 2). ANOVA with prime congruency and probe congruency as factors failed to find a main effect of prime congruency on probe RTs, $F(2,38)=1.94, p=.16$, again replicating the absence of post-conflict slowing in Simon trials. The main effect of probe congruency was significant, $F(1,19)=26.53, M S E=869.23, p<.001$, indicating a Simon effect. Most important, the two-way interaction was also significant, $F(2,38)=8.91, M S E=754.86, p<0.01$, indicating a Gratton effect. The ANOVA on PEs was not significant (prime congruency: $F<1$; probe congruency: $F=2.69, p=.12$; interaction: $F<1)$.

Probe trials: univalent shape discrimination task RTs are shown in Fig. 2d. There was a significant effect of prime congruency on RTs, $F(2,38)=10.85, M S E=505.31$, $p<0.001$. Shape discrimination RTs were similar after congruent and after neutral Simon trials, $t(19)=1.42$, $p=0.17$. However, RTs after incongruent Simon trials were longer than after congruent and neutral Simon trials, both $t$ 's $(19)>3.50$, both $p$ 's $<0.01$. The ANOVA on PEs was not significant $(F<1)$.

Probe trials: univalent location discrimination task RTs are shown in Fig. 2d. There was a significant effect of prime congruency on RTs, $F(2,38)=19.86, M S E=312.17$, $p<0.001$. RTs were similar after congruent and after neutral Simon trials, $t(19)=0.93, p=0.36$. In contrast, location discrimination RTs were longer after incongruent Simon trials than after congruent, $t(19)=5.34, p<.001$, and than after neutral Simon trials, $t(19)=5.73, p<.001$.

In the PEs, there was a main effect of prime congruency, $F(2,38)=18.76, M S E=9.45, p<0.001$, indicating an increase of errors after incongruent Simon trials $(7.3 \%)$, compared to after congruent $(1.6 \%)$ and after neutral trials $(2.7 \%)$.

\section{Discussion}

We observed post-conflict slowing when measured in univalent trials, that is, when a task-focusing component is excluded. This was observed in two experiments, with different relevant dimensions (color and shape) and different mappings (2:2 and 6:2), attesting to the robustness of the effect. Earlier, we have established that subjects slow down after infrequent events (postoddball slowing; Notebaert et al., 2009). In general, it appears that different task problems or interruptions 

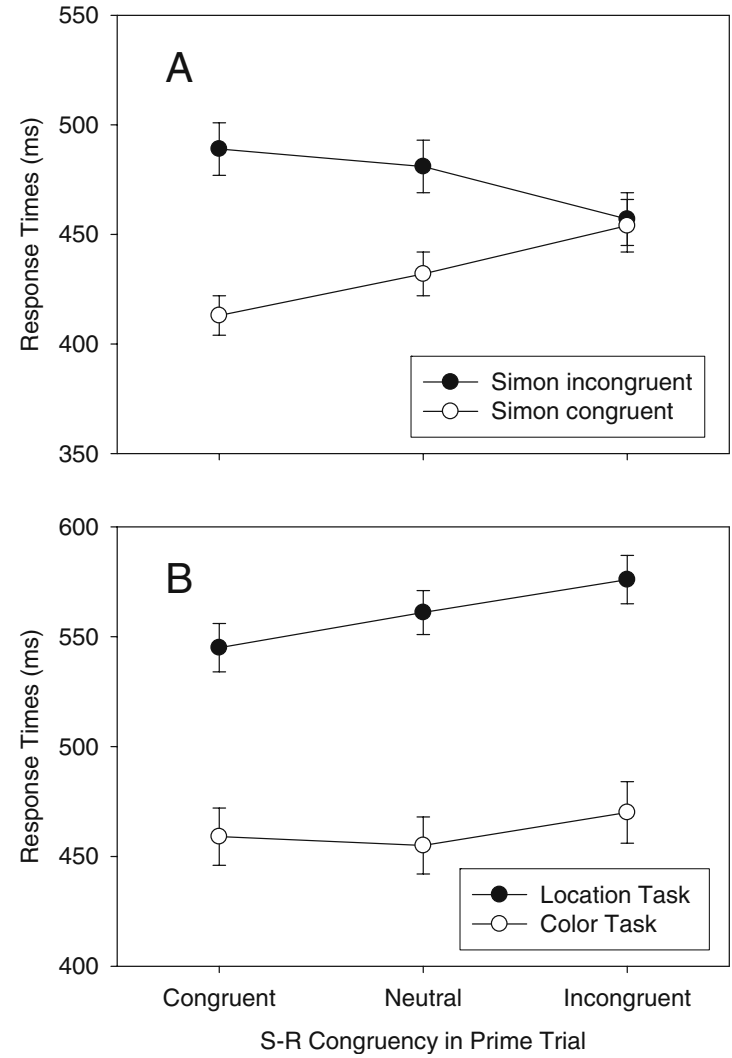

Fig. 2 a, b Data of Experiment 1. a Effect of spatial congruency in the prime-trial Simon task on RTs in the probe-trial Simon task. b Effect of spatial congruency in the prime-trial Simon task on univalent color discrimination and univalent location discrimination tasks. c, d

(errors, incongruencies, infrequent events) lead to a slowing down of task performance.

Reasoning from conflict monitoring theory, we have focused on the similarities between conflict and error processing. However, a consistent application of this framework would seem to predict that there is also no post-error slowing on bivalent (congruent/incongruent) stimuli: If post-conflict focusing masks post-conflict slowing, then should not post-error focusing also mask posterror slowing? Yet, post-error slowing is regularly ob-
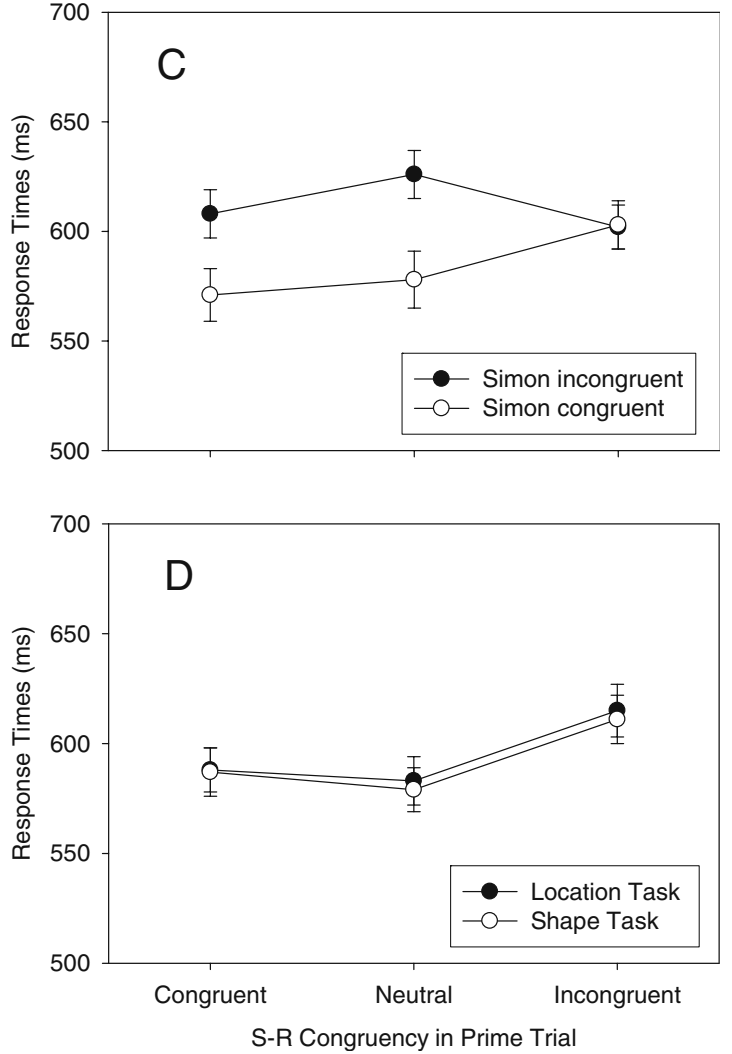

Data of experiment 2. c Effect of spatial congruency in the prime-trial Simon task on RTs in the probe-trial Simon task. d Effect of spatial congruency in the prime-trial Simon task on univalent shape discrimination and univalent location discrimination tasks

served. This difference between conflict and error processing can be illuminated from the point of view of a recent version of conflict monitoring theory proposed by Verguts and Notebaert (2008, 2009; for a similar reasoning see Notebaert \& Verguts, in press). According to this model, conflict adaptation operates by binding active representations more strongly together. On conflict trials, this has the consequence of binding the relevant task demand unit more strongly to the currently relevant input units. On error trials, however, it is much less clear what to predict: Almost by
Table 2 RTs and PEs (in parentheses) in probe trials of experiments 1 and 2

$C$ congruent, $I C$ incongruent

\begin{tabular}{llll}
\hline & \multicolumn{2}{l}{ Congruency in preceding prime trial } \\
\cline { 2 - 4 } & Congruent & Neutral & Incongruent \\
\hline Experiment 1 (Simon C) & $413(0.7)$ & $432(1.2)$ & $454(3.2)$ \\
Experiment 1 (Simon IC) & $489(6.3)$ & $481(5.8)$ & $457(2.6)$ \\
Experiment 1 (color probe) & $459(1.6)$ & $455(1.6)$ & $470(3.7)$ \\
Experiment 1 (location probe) & $545(8.3)$ & $561(12.0)$ & $576(12.5)$ \\
Experiment 2 (Simon C) & $571(3.4)$ & $578(3.3)$ & $603(3.1)$ \\
Experiment 2 (Simon IC) & $608(5.1)$ & $625(5.3)$ & $602(3.8)$ \\
Experiment 2 (shape probe) & $587(2.6)$ & $579(2.6)$ & $611(3.2)$ \\
Experiment 2 (location probe) & $588(1.6)$ & $583(2.7)$ & $615(7.3)$ \\
\hline
\end{tabular}


definition, activations are incorrect on error trials, and because the activation pattern determines the aftereffects, the post-error focusing component should be much less reliable, allowing more opportunity for post-error slowing to show up. Apparently inconsistent with this explanation, post-error focusing has been reported (Ridderinkhof, 2002), but we note that one must be cautious in interpreting this particular result. As noted before, error is naturally correlated with conflict, and error and conflict were not orthogonalized in this study. The existence of post-error focusing hence remains to be investigated further.

When attributing sequence effects to cognitive control processes, one has to be cautious that the sequence effects cannot be described in terms of low-level feature repetition effects. For instance, it has been argued that the Gratton effect can also be explained in terms of priming or feature integration processes (e.g., Hommel, Proctor, \& Vu, 2004; Mayr, Awh, \& Laurey, 2003). Although this could have modulated the Gratton effect on bivalent probe trials, we are confident that post-conflict slowing on univalent trials is not modulated by stimulus repetition or integration effects. In experiment 1 for example, univalent color discrimination trials have the same amount of feature overlap with congruent, incongruent, and neutral prime trials. Hence, this alternative account cannot be applied to post-conflict slowing.

Our findings point to a different aspect of the design that requires caution when investigating cognitive control. This is the fact that different tendencies (in this case, task focusing and slowing) may counteract one another, effectively hiding one (or possibly both). Hence, a methodological advice is to investigate behavioral measures of control with inducer tasks on the one hand (our prime trials), which implement the relevant manipulation (e.g., congruency) and diagnostic tasks on the other hand (our probe trials), which are as simple as possible and are used to measure the dependent variable of interest (cf. Notebaert, Gevers, Verguts, \& Fias, 2006).

Although the RT data were consistent across conditions and experiments, effects in the PEs were either nonsignificant or in the same direction as the RTs (more errors after incongruency). Intuitively, one might think that lowering the activation in the response nodes after conflict functionally corresponds to increasing the boundaries in a diffusion model, so that higher RTs would be associated with lower PEs. Simulations with the conflict-monitoring model show that this is not necessarily the case, however. In particular, when the response activation is low overall, lowering activation in all response units decreases the discriminability between the responses. As an example, suppose two response units have activation of 0.3 and 0.1 , respectively. If an inhibitory term of 0.2 is subtracted from both (reflecting the reduced baseline activation), activations become 0.1 and 0 (if activation cannot go below zero), resulting in a smaller absolute difference between the activation values. A similar argument holds if the lower bound on activation is not exactly zero but some value above zero. Hence, the effect of response inhibition on PEs is not always straightforward and remains to be more fully investigated.

To conclude, this paper confirms that error and conflict can have similar consequences or adaptations, consistent with the predictions of conflict monitoring theory. However, investigating these adaptations is hindered by the fact that different adaptations (task focusing and slowing) will tend to interact on bivalent trials. Hence, appropriate probe trials to investigate cognitive control must be chosen very carefully.

Author Note Tom Verguts and Wim Notebaert, Department of Psychology, Ghent University, Belgium. Wilfried Kunde and Peter Wühr, Institut für Psychologie, Technische Universität Dortmund, Germany. Correspondence can be sent to Tom Verguts, Department of Psychology, H. Dunantlaan 2, Ghent University, Ghent, Belgium. Email tom.verguts@ugent.be.

\section{References}

Botvinick, M. M., Braver, T. S., Barch, D. M., Carter, C. S., \& Cohen, J. D. (2001). Conflict monitoring and cognitive control. Psychological Review, 108, 624-652.

Gratton, G., Coles, M. G. H., \& Donchin, E. (1992). Optimizing the use of information - Strategic control of activation of responses. Journal of Experimental Psychology: General, 121, 480-506.

Hommel, B., Proctor, R. W., \& Vu, K.-P. L. (2004). A featureintegration account of sequential effects in the Simon task. Psychological Research, 68, 1-17.

Kerns, J. G., Cohen, J. D., Macdonald, A. W., Cho, R. Y., Stenger, V. A., \& Carter, C. S. (2004). Anterior cingulate conflict monitoring and adjustments in control. Science, 303, 1023-1026.

Mayr, U., Awh, E., \& Laurey, P. (2003). Conflict adaptation effects in the absence of executive control. Nature Neuroscience, 6, 450 452.

Masson, M. E. J., Bub, D. N., Woodward, T. S., \& Chan, J. C. K. (2003). Modulation of word-reading processes in task switching. Journal of Experimental Psychology: General, 132, 400-418.

Meier, B., Woodward, T. S., Rey-Mermet, A., \& Graf, P. (2009). The bivalency effect in task switching: General and enduring. Canadian Journal of Experimental Psychology, 63, 201-210.

Notebaert, W., \& Verguts, T. (in press). Conflict and error monitoring in the Simon task. Acta Psychologica. doi:10.1016/j.actpsy.2010.05.006

Notebaert, W., Gevers, W., Verguts, T., \& Fias, W. (2006). Shared spatial representations for numbers and space: The reversal of the Simon and SNARC effects. Journal of Experimental Psychology: Human Perception and Performance, 32, 11191207.

Notebaert, W., Houtman, F., Van Opstal, F., Gevers, W., Fias, W., \& Verguts, T. (2009). Post-error slowing: An orienting account. Cognition, 111, 275-279. 
Notebaert, W., \& Verguts, T. (2008). Cognitive control acts locally. Cognition, 106, 1071-1080.

Rabbitt, P. M., \& Phillips, S. (1967). Error-detection and correction latencies as a function of S-R compatibility. The Quarterly Journal of Experimental Psychology, 19, 37-42.

Ridderinkhof, K. R. (2002). Micro- and macro-adjustments of task set: Activation and suppression in conflict tasks. Psychological Research, 66, 312-323.
Ullsperger, M., Bylsma, L. M., \& Botvinick, M. M. (2005). The conflict-adaptation effect: It's not just priming. Cognitive, Affective \& Behavioral Neuroscience, 5, 467-472.

Verguts, T., \& Notebaert, W. (2008). Hebbian learning of cognitive control: Dealing with specific and nonspecific adaptation. Psychological Review, 115, 518-525.

Verguts, T., \& Notebaert, W. (2009). Adaptation by binding: A learning account of cognitive control. Trends in Cognitive Sciences, 13, 252-257. 